\title{
Existence results for a general class of sequential hybrid fractional differential equations
}

\author{
Rahmat Ali Khan ${ }^{1,2}$, Shaista Gul ${ }^{1}$, Fahd Jarad ${ }^{3,4^{*}}$ and Hasib Khan ${ }^{2 *}$
}

"Correspondence:

fahd@cankaya.edu.tr; hasibkhan13@yahoo.com

${ }^{3}$ Department of Mathematics, Cankaya University, Ankara, Turkey

2Department of Mathematics,

Shaheed Benazir Bhutto University,

Sheringal Dir Upper, Khyber

Pakhtunkhwa, Pakistan

Full list of author information is

available at the end of the article

\section{Springer}

\begin{abstract}
In this paper, we study a class of nonlinear boundary value problems (BVPS) consisting of a more general class of sequential hybrid fractional differential equations (SHFDEs) together with a class of nonlinear boundary conditions at both end points of the domain. The nonlinear functions involved depend explicitly on the fractional derivatives. We study the necessary conditions required for the unique solution to the suggested BVP under the Caratheodory conditions using the technique of measure of noncompactness and degree theory. We also develop conditions for uniqueness results and also on stability analysis.
\end{abstract}

MSC: 34A08; 35R 11

Keywords: Boundary value problems; Sequential hybrid fractional differential equations; Three point boundary conditions; Existence and uniqueness results; Stability analysis

\section{Introduction}

The existence theory for solutions of BVPs of hybrid fractional differential equations and SHFDEs has attracted the attention of many researchers, we refer to [1-11] and the references therein for the recent development in this particular area of interest. In most of these studies, BVPs with lower order fractional derivatives together with either constant or linear boundary conditions are considered. However, in many situations, there are possibilities to have nonlinear conditions at the boundary, and the differential equations may be of higher order involving functions that depend explicitly on the fractional order derivatives. For example, in case of head flow problems, there are possibilities to have some source or sink on both sides of the boundary (at $x=0$ and $x=1$ ) which may be nonlinear functions and a controller at $x=\zeta_{0}\left(0<\zeta_{0}<1\right)$. Such situation may have importance in application point of view and also in theoretical development. The purpose of this paper is to investigate existence results for BVPs involving nonlinear boundary conditions at both end points, that is, we study the following class of three point

(c) The Author(s) 2021. This article is licensed under a Creative Commons Attribution 4.0 International License, which permits use sharing, adaptation, distribution and reproduction in any medium or format, as long as you give appropriate credit to the original author(s) and the source, provide a link to the Creative Commons licence, and indicate if changes were made. The images or other third party material in this article are included in the article's Creative Commons licence, unless indicated otherwise in a credit line to the material. If material is not included in the article's Creative Commons licence and your intended use is not permitted by statutory regulation or exceeds the permitted use, you will need to obtain permission directly from the copyright holder. To view a copy of this licence, visit http://creativecommons.org/licenses/by/4.0/. 
BVPs:

$$
\begin{aligned}
& { }^{c} \mathcal{D}^{\vartheta}\left[\frac{{ }^{c} \mathcal{D}^{\omega} u(t)-\sum_{1}^{m} I^{\beta_{i}} h_{i}\left(t, u(t), \mathcal{D}^{\omega-1} u(t)\right)}{f\left(t, u(t), \mathcal{D}^{\omega-1} u(t)\right)}\right]=g\left(t, u(t), I^{\gamma} u(t)\right), \quad t \in \mathrm{I}=[0,1] \\
& { }^{c} \mathcal{D}^{\omega} u(0)=0, \quad u(0)=\psi_{1}\left(u\left(\zeta_{0}\right)\right), \quad u(1)=\psi_{2}\left(u\left(\zeta_{0}\right)\right),
\end{aligned}
$$

where the parameters are such that $0<\vartheta \leq 1,1<\omega \leq 2,0<\zeta_{0}<1$, the functions $f: \mathrm{I} \times \mathcal{R}_{e} \times \mathcal{R}_{e} \rightarrow \mathcal{R}_{e}-\{0\}, h_{i}: \mathrm{I} \times \mathcal{R}_{e} \times \mathcal{R}_{e} \rightarrow \mathcal{R}_{e}(i=1,2, \ldots, m)$, and $g: \mathrm{I} \times \mathcal{R}_{e} \times \mathcal{R}_{e} \rightarrow \mathcal{R}_{e}$ satisfy the Caratheodory conditions, the boundary functions $\psi_{1}, \psi_{2}: \mathcal{R}_{e} \rightarrow \mathcal{R}_{e}$ are nonlinear, and $\mathcal{R}_{e}$ represents the set of real numbers. To the best of our knowledge, existence, uniqueness, and stability results have never been studied for BVP (1) previously.

Choose $\Omega_{0}$ a bounded subset of a Banach space $\mathbb{E}$, where $\mathbb{E}=\left\{u \in C(\mathrm{I}): \mathcal{D}^{\omega-1} u \in C(\mathrm{I})\right\}$, endowed with the norm $\|u\|_{1}=\max _{0 \leq t \leq 1}|u(t)|+\max _{0 \leq t \leq 1}\left|\mathcal{D}^{\omega-1} u\right|$. We recall the following definition [12].

\section{Preliminaries}

Definition 2.1 The Kuratowski measure of noncompactness $\varrho^{* *}: \mathbb{S} \rightarrow[0, \infty)$ of a set $\mathbb{S} \subseteq$ $\mathbb{E}$ is defined as

$$
\varrho^{* *}(\mathbb{S})=\inf \{d>0: \mathbb{S} \in \mathbb{B} \text { admits a finite cover by sets of diameter } \leq d\},
$$

where $\mathbb{B}$ denotes the family of all bounded subsets of $\mathbb{E}$.

Recall the following definitions and propositions from [13].

Proposition 2.1 The Kuratowski measure $\varrho^{* *}$ satisfies the following properties:

(i) $\varrho^{* *}(\mathbb{S})=0$ iff $\mathbb{S}$ is relatively compact;

(ii) $\varrho^{* *}$ is a seminorm, i.e., $\varrho^{* *}\left(\lambda^{* *} \mathbb{S}\right)=\left|\lambda^{* *}\right| \varrho^{* *}(\mathbb{S}), \lambda^{* *} \in \mathbb{R}$, and

$$
\varrho^{* *}\left(\mathbb{S}_{1}+\mathbb{S}_{2}\right) \leq \varrho^{* *}\left(\mathbb{S}_{1}\right)+\varrho^{* *}\left(\mathbb{S}_{2}\right) ;
$$

(iii) $\mathbb{S}_{1} \subset \mathbb{S}_{2}$ implies $\varrho^{* *}\left(\mathbb{S}_{1}\right) \leq \varrho^{* *}\left(\mathbb{S}_{2}\right) ; \varrho^{* *}\left(\mathbb{S}_{1} \cup \mathbb{S}_{2}\right)=\max \left\{\varrho^{* *}\left(\mathbb{S}_{1}\right), \varrho^{* *}\left(\mathbb{S}_{2}\right)\right\}$;

(iv) $\varrho^{* *}(\overline{\mathbb{S}})=\varrho^{* *}(\operatorname{conv} \mathbb{S})=\varrho^{* *}(\mathbb{S})$.

Definition 2.2 Let the function $T: \mathbb{S} \rightarrow \mathbb{E}$ be continuous and bounded, where $\mathbb{S} \subset \mathbb{E}$. Then $T$ is $\varrho^{* *}$-Lipschitz ( $\mathrm{k}$-set contraction) if there exists $k \geq 0$ such that

$$
\varrho^{* *}\left(T\left(\Omega_{0}\right)\right) \leq k \varrho^{* *}\left(\Omega_{0}\right), \quad \forall \text { bounded } \Omega_{0} \subset \mathbb{S} .
$$

Furthermore, if $k<1$, then $T$ will be a strict $\varrho^{* *}$-contraction.

Definition 2.3 $T$ is said to be $\varrho^{* *}$-condensing if

$$
\varrho^{* *}\left(T\left(\Omega_{0}\right)\right)<\varrho^{* *}\left(\Omega_{0}\right), \quad \forall \text { bounded } \Omega_{0} \subset \mathbb{S} \text { with } \varrho^{* *}\left(\Omega_{0}\right)>0 .
$$

In other words, $\varrho^{* *}\left(T\left(\Omega_{0}\right)\right) \geq \varrho^{* *}\left(\Omega_{0}\right)$ implies $\varrho^{* *}\left(\Omega_{0}\right)=0$.

Proposition 2.2 For $\varrho^{* *}$-Lipschitz maps $\mathbb{A}, \mathbb{B}: \Omega_{0} \rightarrow \mathbb{E}$ with constants $k$ and $k^{\prime}$, respectively, $\mathbb{A}+\mathbb{B}: \Omega_{0} \rightarrow \mathbb{E}$ is $\varrho^{* *}$-Lipschitz with constant $k+k^{\prime}$. 
Proposition 2.3 Let $\mathbb{T}: \Omega_{0} \rightarrow \mathbb{E}$ be compact, then $\mathbb{T}$ is $\varrho^{* *}$-Lipschitz with constant $K=0$.

Proposition 2.4 If $\mathbb{T}: \Omega_{0} \rightarrow \mathbb{E}$ satisfies Lipschitz with constant $k$, then $\mathbb{T}$ is $\varrho^{* *}$-Lipschitz with the same constant $k$.

The following theorem [14] will be used in the sequel.

Theorem 2.4 Let $\mathbb{T}: \mathbb{E} \rightarrow \mathbb{E}$ be a $\varrho^{* *}$ condensing map and

$$
\Theta=\left\{u \in \mathbb{E}: \exists \lambda^{* *} \in[0,1] \text { s.t } u=\lambda^{* *} \mathbb{T} u\right\} .
$$

If $\Theta$ is a bounded set in $\mathbb{E}$, that is, there exists $r>0$ such that $\Theta \subset B_{r}(0)$, then the topological degree

$$
\mathbb{D}\left(I-\lambda^{* *} \mathbb{T}, B_{r}(0), 0\right)=1, \quad \forall \lambda^{* *} \in[0,1],
$$

that is, $\mathbb{T}$ has a fixed point in $B_{r}(0)$.

Organization of the paper. This article consists of five sections. The first section explains the importance of the article and the related literature. In the second section, we study sufficient conditions for the existence and uniqueness of solutions to the hybrid fractional differential equations (1). Third section is reserved for the Hyers-Ulam stability of problem (1). Section 4 explains the application of the results, and finally the conclusion of the article is given in Sect. 5 .

\section{Existence criteria}

This section of the article is reserved for the existence and uniqueness of solution of hybrid problem (1) with the help of the fixed point approach. For these, we first transform the suggested problem into an integral form of the problem.

Lemma 3.1 For integrable functions $f$, $g$, and $h_{i}$ on I, problem (1) has an integral representation given by

$$
\begin{aligned}
u(t)= & \int_{0}^{1}\left(\sum_{1}^{m} K_{\beta_{i}}(s, t) h_{i}\left(s, u(s), \mathcal{D}^{\omega-1} u(s)\right)+K_{0}(s, t) \Psi\left(s, u(s), \mathcal{D}^{\omega-1} u(s)\right)\right) d s \\
& +t \psi_{2}\left(u\left(\zeta_{0}\right)\right)+(1-t) \psi_{1}\left(u\left(\zeta_{0}\right)\right),
\end{aligned}
$$

where $\Psi\left(t, u(t), \mathcal{D}^{\omega-1} u(t)\right)=f\left(t, u(t), \mathcal{D}^{\omega-1} u(t)\right) I^{\vartheta} g\left(t, u(t), I^{\gamma} u(t)\right)$, and

$$
\begin{aligned}
& K_{\beta_{i}}(s, t)=\frac{-1}{\Gamma\left(\omega+\beta_{i}\right)} \begin{cases}t(1-s)^{\omega-1+\beta_{i} ;} & t \leq s, \\
t(1-s)^{\omega-1+\beta_{i}}-(t-s)^{\omega-1+\beta_{i} ;} & s \leq t,\end{cases} \\
& K_{0}(s, t)=\frac{-1}{\Gamma(\omega)} \begin{cases}t(1-s)^{\omega-1} ; & t \leq s, \\
t(1-s)^{\omega-1}-(t-s)^{\omega-1} ; & s \leq t .\end{cases}
\end{aligned}
$$


Proof Applying the $\vartheta$ th integral $\left(I^{\vartheta}\right)$ to both sides of (1), we obtain

$$
{ }^{c} \mathcal{D}^{\omega} u(t)-\sum_{1}^{m} I^{\beta_{i}} h_{i}\left(t, u(t), \mathcal{D}^{\omega-1} u(t)\right)=f\left(t, u(t), \mathcal{D}^{\omega-1} u(t)\right) I^{\vartheta} g\left(t, u(t), I^{\gamma} u(t)\right)+C_{1} .
$$

The initial condition ${ }^{c} \mathcal{D}^{\omega} u(0)=0$ results in $C_{1}=0$, and hence we obtain

$$
\begin{aligned}
{ }^{c} \mathcal{D}^{\omega} u(t) & =\sum_{1}^{m} I^{\beta_{i}} h_{i}\left(t, u(t), \mathcal{D}^{\omega-1} u(t)\right)+f\left(t, u(t), \mathcal{D}^{\omega-1} u(t)\right) I^{\vartheta} g\left(t, u(t), I^{\gamma} u(t)\right) \\
& =\sum_{1}^{m} I^{\beta_{i}} h_{i}\left(t, u(t), \mathcal{D}^{\omega-1} u(t)\right)+\Psi\left(t, u(t), \mathcal{D}^{\omega-1} u(t)\right),
\end{aligned}
$$

where $\Psi\left(t, u(t), \mathcal{D}^{\omega-1} u(t)\right)=f\left(t, u(t), \mathcal{D}^{\omega-1} u(t)\right) I^{\vartheta} g\left(t, u(t), I^{\gamma} u(t)\right)$. Applying the $\omega$ th integral $\left(I^{\omega}\right)$ on (5) and using the semigroup property of the integrals, we obtain

$$
u(t)=\sum_{1}^{m} I^{\omega+\beta_{i}} h_{i}\left(t, u(t), \mathcal{D}^{\omega-1} u(t)\right)+I^{\omega} \Psi\left(t, u(t), \mathcal{D}^{\omega-1} u(t)\right)+D_{1}+D_{2} t
$$

The boundary conditions $u(0)=\psi_{1}\left(u\left(\zeta_{0}\right)\right), u(1)=\psi_{2}\left(u\left(\zeta_{0}\right)\right)$ respectively give $D_{1}=$ $\psi_{1}\left(u\left(\zeta_{0}\right)\right)$ and

$$
D_{2}=\psi_{2}\left(u\left(\zeta_{0}\right)\right)-\psi_{1}\left(u\left(\zeta_{0}\right)\right)-I^{\omega} \Psi\left(1, u(1), \mathcal{D}^{\omega-1} u(1)\right)-\sum_{1}^{m} I^{\omega+\beta_{i}} h_{i}\left(1, u(1), \mathcal{D}^{\omega-1} u(1)\right)
$$

where $I^{\omega} \psi\left(1, u(1), \mathcal{D}^{\omega-1} u(1)\right)$ denotes the value of the integral $I^{\omega} \Psi\left(t, u(t), \mathcal{D}^{\omega-1} u(t)\right)$ at $t=$ 1 and $I^{\omega+\beta_{i}} h_{i}\left(1, u(1), \mathcal{D}^{\omega-1} u(1)\right)$ denotes the value of the integral $I^{\omega+\beta_{i}} h_{i}\left(t, u(t), \mathcal{D}^{\omega-1} u(t)\right)$ at $t=1$ for $i=1,2,3, \ldots m$. Hence, it follows that

$$
\begin{aligned}
u(t)= & \sum_{1}^{m} I^{\omega+\beta_{i}} h_{i}\left(t, u(t), \mathcal{D}^{\omega-1} u(t)\right)+I^{\omega} \Psi\left(t, u(t), \mathcal{D}^{\omega-1} u(t)\right)+\psi_{1}\left(u\left(\zeta_{0}\right)\right)+t\left(\psi_{2}\left(u\left(\zeta_{0}\right)\right)\right. \\
& -\psi_{1}\left(u\left(\zeta_{0}\right)\right)-I^{\omega} \Psi\left(1, u(1), \mathcal{D}^{\omega-1} u(1)\right)-\sum_{1}^{m} I^{\omega+\beta_{i}} h_{i}\left(1, u(1), \mathcal{D}^{\omega-1} u(1)\right) \\
= & \sum_{1}^{m}\left(I^{\omega+\beta_{i}} h_{i}\left(t, u(t), \mathcal{D}^{\omega-1} u(t)\right)-t I^{\omega+\beta_{i}} h_{i}\left(1, u(1), \mathcal{D}^{\omega-1} u(1)\right)\right. \\
& +I^{\omega} \Psi\left(t, u(t), \mathcal{D}^{\omega-1} u(t)\right)-t I^{\omega} \Psi\left(1, u(1), \mathcal{D}^{\omega-1} u(1)\right)+t \psi_{2}\left(u\left(\zeta_{0}\right)\right) \\
& +(1-t) \psi_{1}\left(u\left(\zeta_{0}\right)\right)
\end{aligned}
$$

which can be rewritten as

$$
\begin{aligned}
u(t)= & \int_{0}^{1}\left[\sum_{1}^{m} K_{\beta_{i}}(s, t) h_{i}\left(s, u(s), \mathcal{D}^{\omega-1} u(s)\right)+K_{0}(s, t) \Psi\left(s, u(s), \mathcal{D}^{\omega-1} u(s)\right)\right] d s \\
& +(1-t) \psi_{1}\left(u\left(\zeta_{0}\right)\right)+t \psi_{2}\left(u\left(\zeta_{0}\right)\right) .
\end{aligned}
$$


From (7), it follows that

$$
\begin{aligned}
\mathcal{D}^{\omega-1} u(t)= & \int_{0}^{1}\left[\sum_{1}^{m} G_{\beta_{i}}(s, t) h_{i}\left(s, u(s), \mathcal{D}^{\omega-1} u(s)\right)+G_{0}(s, t) \Psi\left(s, u(s), \mathcal{D}^{\omega-1} u(s)\right)\right] d s \\
& +\frac{t^{2-\omega}}{\Gamma(3-\omega)}\left(\psi_{2}\left(u\left(\zeta_{0}\right)\right)-\psi_{1}\left(u\left(\zeta_{0}\right)\right),\right.
\end{aligned}
$$

where

$$
\begin{aligned}
G_{\beta_{i}}(s, t) & =\mathcal{D}^{\omega-1} K_{\beta_{i}}(s, t) \\
& =\frac{-1}{\Gamma(3-\omega) \Gamma\left(\omega+\beta_{i}\right)} \begin{cases}t^{2-\omega}(1-s)^{\omega-1+\beta_{i}} ; & t \leq s, \\
t^{2-\omega}(1-s)^{\omega-1+\beta_{i}}-(t-s)^{\beta_{i}} ; & s \leq t,\end{cases} \\
G_{0}(s, t) & =\mathcal{D}^{\omega-1} K_{0}(s, t)=\frac{-1}{\Gamma(3-\omega) \Gamma(\omega)} \begin{cases}t^{2-\omega}(1-s)^{\omega-1} ; & t \leq s, \\
t^{2-\omega}(1-s)^{\omega-1}-\Gamma \omega ; & s \leq t .\end{cases}
\end{aligned}
$$

From (3), (4), (9), and (10), it follows that

$$
\begin{aligned}
& \max _{t \in[0,1]}\left|K_{\beta_{i}}(s, t)\right|=\frac{s(1-s)^{\omega-1+\beta_{i}}}{\Gamma\left(\omega+\beta_{i}\right)} \leq \frac{1}{\Gamma\left(\omega+\beta_{i}\right)}, \\
& \max _{t \in[0,1]}\left|K_{0}(s, t)\right|=\frac{s(1-s)^{\omega-1}}{\Gamma(\omega)} \leq \frac{1}{\Gamma(\omega)}, \\
& \max _{t \in[0,1]}\left|G_{\beta_{i}}(s, t)\right| \leq \frac{1}{\Gamma(3-\omega) \Gamma\left(\omega+\beta_{i}\right)}, \\
& \max _{t \in[0,1]}\left|G_{0}(s, t)\right| \leq \frac{1}{\Gamma(3-\omega) \Gamma(\omega)} .
\end{aligned}
$$

Define operators $\mathbb{A}, \mathbb{B}: E=C\left(\mathrm{I}, \mathcal{R}_{e}\right) \rightarrow E$ by

$$
\begin{aligned}
& \mathbb{A}(u)=\int_{0}^{1}\left(\sum_{1}^{m} K_{\beta_{i}}(s, t) h_{i}\left(s, u(s), \mathcal{D}^{\omega-1} u(s)\right)+K_{0}(s, t) \Psi\left(s, u(s), \mathcal{D}^{\omega-1} u(s)\right)\right) d s, \\
& \mathbb{B}(u)=(1-t) \psi_{1}\left(u\left(\zeta_{0}\right)\right)+t \psi_{2}\left(u\left(\zeta_{0}\right)\right),
\end{aligned}
$$

then (7) takes the form of the operator equation

$$
u(t)=\mathbb{A} u(t)+\mathbb{B} u(t)=\mathbb{T} u(t), \quad t \in \mathrm{I},
$$

and fixed points of operator equation (13) are solutions of BVP (1). Now, we list the following hypotheses.

$\left(H_{1}\right) f: \mathrm{I} \times \mathcal{R}_{e} \times \mathcal{R}_{e} \rightarrow \mathcal{R}_{e}-\{0\}, h_{i}: \mathrm{I} \times \mathcal{R}_{e} \times \mathcal{R}_{e} \rightarrow \mathcal{R}_{e}(i=1,2, \ldots, m)$, and $g: \mathrm{I} \times \mathcal{R}_{e} \times$ $\mathcal{R}_{e} \rightarrow \mathcal{R}_{e}$ satisfy the Caratheodory conditions.

$\left(H_{2}\right)$ There exist positive constants $k_{1}, k_{2} \in[0,1), q \in(0,1)$, and $d_{1}, d_{2}, e_{1}, e_{2}$ such that, for $u, u_{1}, u_{2} \in E$, we have

$$
\begin{array}{ll}
\left|\psi_{1}\left(u_{2}\right)-\psi_{1}\left(u_{1}\right)\right| \leq k_{1}\left|u_{2}-u_{1}\right|, & \left|\psi_{1}(u)\right| \leq e_{1}|u|^{q}+e_{2}, \\
\left|\psi_{2}\left(u_{2}\right)-\psi_{2}\left(u_{1}\right)\right| \leq k_{2}\left|u_{2}-u_{1}\right|, & \left|\psi_{2}(u)\right| \leq d_{1}|u|^{q}+d_{2} .
\end{array}
$$


$\left(H_{3}\right)$ There exist positive continuous functions $\varrho^{* *}, \rho: \mathrm{I} \rightarrow \mathcal{R}_{e}$, parameters $0<q$, $\delta<1$, and positive constants $\theta_{i}, v, \xi$ such that, for $u \in E$,

$$
\begin{aligned}
& \left|h_{i}\left(t, u(t), \mathcal{D}^{\omega-1} u(t)\right)\right| \leq \theta_{i}, \\
& \left|f\left(t, u(t), \mathcal{D}^{\omega-1} u(t)\right)\right| \leq \varrho^{* *}(t)\left(|u(t)|+\left|\mathcal{D}^{\omega-1} u(t)\right|\right)^{\delta}+\xi, \\
& \left|g\left(t, u(t), I^{\gamma} u(t)\right)\right| \leq|\rho(t)|+v\left(|u|^{q}+\left|I^{\gamma} u\right|^{q}\right) .
\end{aligned}
$$

$\left(H_{4}\right)$ There exist positive constants $\lambda^{* *}{ }_{i}$ for $i=1,2, \ldots, m$ such that, for $u, \bar{u} \in E$ and $\varrho^{* *}{ }_{0}=$ $\max _{t \in I} Q^{* *}(t), \rho_{0}=\max _{t \in I} \rho(t)$

$$
\begin{aligned}
& \left|h_{i}\left(t, u(t), \mathcal{D}^{\omega-1} u(t)\right)-h_{i}\left(t, \bar{u}(t), \mathcal{D}^{\omega-1} \bar{u}(t)\right)\right| \\
& \quad \leq \lambda^{* *}{ }_{i}\left(|u-\bar{u}|+\left|\mathcal{D}^{\omega-1} u-\mathcal{D}^{\omega-1} \bar{u}\right|\right), \\
& \left|f\left(t, u(t), \mathcal{D}^{\omega-1} u(t)\right)-f\left(t, \bar{u}(t), \mathcal{D}^{\omega-1} \bar{u}(t)\right)\right| \\
& \quad \leq \varrho^{* *}{ }_{0}\left(|u-\bar{u}|+\left|\mathcal{D}^{\omega-1} u-\mathcal{D}^{\omega-1} \bar{u}\right|\right), \\
& \left|g\left(t, u(t), \mathcal{I}^{\gamma} u(t)\right)-g\left(t, \bar{u}(t), \mathcal{I}^{\gamma} \bar{u}(t)\right)\right| \leq \rho_{0}|u-\bar{u}| .
\end{aligned}
$$

Lemma 3.2 Under condition $\left(H_{2}\right)$, the operator $\mathbb{B}$ is $\varrho^{* *}$-Lipschitz with constant $k=$ $\max \left\{\left(1-t+\frac{t^{2-\omega}}{\Gamma(3-\omega)} k_{1}+\left(t+\frac{t^{2-\omega}}{\Gamma(3-\omega)}\right) k_{2}, t \in \mathrm{I}\right\}\right.$. Further, $\mathbb{B}$ satisfies the following growth condition:

$$
\|\mathbb{B} u(t)\|_{1} \leq d\|u\|_{1}^{q}+e
$$

where $d=\max \left\{\left(t+\frac{t^{2-\omega}}{\Gamma(3-\omega)}\right) d_{1}+\left(1-t+\frac{t^{2-\omega}}{\Gamma(3-\omega)}\right) e_{1}, t \in \mathrm{I}\right\}, e=\max \left\{\left(t+\frac{t^{2-\omega}}{\Gamma(3-\omega)}\right) d_{2}+(1-t+\right.$ $\left.\left.\frac{t^{2-\omega}}{\Gamma(3-\omega)}\right) e_{2}, t \in \mathrm{I}\right\}$.

Proof For $u_{1}, u_{2} \in E$ such that $u_{1}<u_{2}$, using $\left(H_{2}\right)$, we obtain

$$
\begin{aligned}
&\left|\mathbb{B}\left(u_{1}\right)-\mathbb{B}\left(u_{2}\right)\right| \leq(1-t)\left|\psi_{1}\left(u_{1}\left(\zeta_{0}\right)\right)-\psi_{1}\left(u_{2}\left(\zeta_{0}\right)\right)\right|+t\left|\psi_{2}\left(u_{1}\left(\zeta_{0}\right)\right)-\psi_{2}\left(u_{2}\left(\zeta_{0}\right)\right)\right| \\
&\left.\left.\leq(1-t) k_{1} \mid u_{1}\left(\zeta_{0}\right)\right)-u_{2}\left(\zeta_{0}\right)\left|+t k_{2}\right| u_{1}\left(\zeta_{0}\right)\right)-u_{2}\left(\zeta_{0}\right) \mid \\
&\left.=\left((1-t) k_{1}+t k_{2}\right) \mid u_{1}\left(\zeta_{0}\right)\right)-u_{2}\left(\zeta_{0}\right) \mid \\
&\left|\mathcal{D}^{\omega-1} \mathbb{B}\left(u_{1}\right)-\mathcal{D}^{\omega-1} \mathbb{B}\left(u_{2}\right)\right| \\
& \leq \frac{t^{2-\omega}}{\Gamma(3-\omega)}\left|\psi_{1}\left(u_{1}\left(\zeta_{0}\right)\right)-\psi_{1}\left(u_{2}\left(\zeta_{0}\right)\right)\right|+\left|\psi_{2}\left(u_{1}\left(\zeta_{0}\right)\right)-\psi_{2}\left(u_{2}\left(\zeta_{0}\right)\right)\right| \\
&\left.\quad \leq t^{2-\omega} \frac{k_{1}+k_{2}}{\Gamma(3-\omega)} \mid u_{1}\left(\zeta_{0}\right)\right)-u_{2}\left(\zeta_{0}\right) \mid .
\end{aligned}
$$

Hence, from (15) and (16), it follows that

$$
\left\|\mathbb{B}\left(u_{1}\right)-\mathbb{B}\left(u_{2}\right)\right\|_{1} \leq k\left\|u_{1}-u_{2}\right\| \leq k\left\|u_{1}-u_{2}\right\|_{1} .
$$


By Proposition $2.4, \mathbb{B}$ is $\varrho^{* *}$-Lipschitz with constant $k$. Further,

$$
\begin{aligned}
|\mathbb{B}(u)| & \leq(1-t)\left|\psi_{1}\left(u\left(\zeta_{0}\right)\right)\right|+t\left|\psi_{2}\left(u\left(\zeta_{0}\right)\right)\right| \\
& \leq(1-t)\left(d_{1}|u|^{q}+d_{2}\right)+t\left(e_{1}|u|^{q}+e_{2}\right) \\
& =\left(t d_{1}+(1-t) e_{1}\right)|u|^{q}+\left(t d_{2}+(1-t) e_{2}\right), \\
\left|\mathcal{D}^{\omega-1} \mathbb{B}(u)\right| & \leq \frac{t^{2-\omega}}{\Gamma(3-\omega)}\left(\left|\psi_{1}\left(u\left(\zeta_{0}\right)\right)\right|+\left|\psi_{2}\left(u\left(\zeta_{0}\right)\right)\right|\right. \\
& \leq \frac{t^{2-\omega}}{\Gamma(3-\omega)}\left(\left(d_{1}+e_{1}\right)|u|^{q}+\left(d_{2}+e_{2}\right)\right) .
\end{aligned}
$$

From (18) and (19), it follows that

$$
\| \mathbb{B}\left(u\left\|_{1} \leq d\right\| u\left\|^{q}+e \leq d\right\| u \|_{1}^{q}+e\right.
$$

Lemma 3.3 Under conditions $\left(H_{1}\right)$ and $\left(H_{3}\right)$, the operator $\mathbb{A}$ is $\varrho^{* *}$-Lipschitz with zero constant. Further $\mathbb{A}$ satisfies the following growth condition:

$$
\|\mathbb{A} u(t)\| \leq \varepsilon_{0}+£_{1}\|u\|_{1}^{\delta}+£_{2}\|u\|_{1}^{q}+E_{3}\|u\|_{1}^{q+\delta}, \quad u \in \mathbb{E}
$$

Proof By $\left(H_{1}\right)$, the continuity of $h_{i}, \Psi$ with respect to $u$ for each fixed $t \in \mathrm{I}$ implies the continuity of the operator $\mathbb{A}$ for each fixed $t \in I$. Moreover, for each $u \in E$, using $\left(H_{3}\right)$, we obtain

$$
\begin{aligned}
& \left|f\left(t, u(t), \mathcal{D}^{\omega-1} u(t)\right)\right| \leq \varrho^{* *}(t)\|u\|^{\delta}+\xi \\
& \left|g\left(t, u, I^{\gamma} u\right)\right| \leq|\rho(t)|+v\left(\|u\|^{q}+\left\|I^{\gamma} u\right\|^{q}\right) .
\end{aligned}
$$

Hence it follows that

$$
\begin{aligned}
& \left|\Psi\left(t, u(t), \mathcal{D}^{\omega-1} u(t)\right)\right| \\
& =\left|f\left(t, u(t), \mathcal{D}^{\omega-1} u(t)\right)\right|\left|I^{\vartheta} g\left(t, u, I^{\gamma} u\right)\right| \\
& \leq \frac{1}{\Gamma(\vartheta+1)}\left[\varrho^{* *}{ }_{0} \rho_{0}\|u\|^{\delta}\right. \\
& \left.\quad+\varrho^{* *}{ }_{0} v\left(1+\frac{1}{(\Gamma(\gamma+1))^{q}}\right)\|u\|^{\delta+q}+\xi v\left(1+\frac{1}{(\Gamma(\gamma+1))^{q}}\right)\|u\|^{q}+\xi \rho_{0}\right],
\end{aligned}
$$

where $\varrho^{* *}{ }_{0}=\max _{t \in \mathrm{I}}\left|\varrho^{* *}(t)\right|, \rho_{0}=\max _{t \in \mathrm{I}}|\rho(t)|$. Thus $\mathbb{A}$ satisfies the following growth condition:

$$
\begin{aligned}
|\mathbb{A} u(t)|+\left|\mathcal{D}^{\omega-1} \mathbb{A} u(t)\right| \leq & \int_{0}^{1} \sum_{1}^{m}\left(\left|K_{\beta_{i}}(s, t)\right|+\left|G_{\beta_{i}}(s, t)\right|\left|h_{i}\left(s, u(s), \mathcal{D}^{\omega-1} u(s)\right)\right|\right. \\
& +\left(\left|K_{0}(s, t)\right|+\left|G_{0}(s, t)\right|\left|\Psi\left(s, u(s), \mathcal{D}^{\omega-1} u(s)\right)\right| d s\right.
\end{aligned}
$$


which in view of (11) and (21) implies that

$$
\begin{aligned}
|\mathbb{A} u(t)|+\left|\mathcal{D}^{\omega-1} \mathbb{A} u(t)\right| & \\
\leq & \left(1+\frac{1}{\Gamma(3-\omega)}\right)\left(\sum_{1}^{m} \frac{\left\|\theta_{i}\right\|}{\Gamma\left(\omega+\beta_{i}\right)}+\frac{1}{\Gamma(\omega)}\left\|\Psi\left(s, u(s), \mathcal{D}^{\omega-1} u(s)\right)\right\|\right) \\
\leq & \left(1+\frac{1}{\Gamma(3-\omega)}\right)\left(\sum_{1}^{m} \frac{\left\|\theta_{i}\right\|}{\Gamma\left(\omega+\beta_{i}\right)}+\frac{1}{\Gamma(\vartheta+1) \Gamma(\omega)}\left(\varrho^{* *}{ }_{0} \rho_{0}\|u\|^{\delta}\right.\right. \\
& \left.+\varrho^{* *}{ }_{0} \nu\left(1+\frac{1}{(\Gamma(\gamma+1))^{q}}\right)\|u\|^{\delta+q}+\xi \nu\left(1+\frac{1}{(\Gamma(\gamma+1))^{q}}\right)\|u\|^{q}+\xi \rho_{0}\right)
\end{aligned}
$$

Hence, it follows that

$$
\begin{aligned}
\|\mathbb{A} u(t)\|_{1} & \leq \varepsilon_{0}+£_{1}\|u\|^{\delta}+£_{2}\|u\|^{q}+£_{3}\|u\|^{q+\delta} \\
& \leq \varepsilon_{0}+£_{1}\|u\|_{1}^{\delta}+£_{2}\|u\|_{1}^{q}+£_{3}\|u\|_{1}^{q+\delta}, \quad u \in \mathbb{E},
\end{aligned}
$$

where $\ell_{0}=\left(1+\frac{1}{\Gamma(3-\omega)}\right)\left(\sum_{1}^{m} \frac{\left\|\theta_{i}\right\|}{\Gamma\left(\omega+\beta_{i}\right)}+\frac{\rho_{0} \xi}{\Gamma(\omega) \Gamma(\vartheta+1)}\right), £_{1}=\left(1+\frac{1}{\Gamma(3-\omega)}\right) \frac{\varrho^{* *}{ }_{0} \rho_{0}}{\Gamma(\omega) \Gamma(\vartheta+1)}, \underbrace{}_{2}=\frac{\nu \xi}{\Gamma(\omega) \Gamma(\vartheta+1)}(1+$ $\left.\frac{1}{\Gamma(3-\omega)}\right)\left(1+\frac{1}{(\Gamma(\gamma+1))^{q}}\right)$, and $E_{3}=\frac{\nu \varrho^{* *} 0}{\Gamma(\omega) \Gamma(\vartheta+1)}\left(1+\frac{1}{\Gamma(3-\omega)}\right)\left(1+\frac{1}{(\Gamma(\gamma+1))^{q}}\right)$. From (23), it also follows that $\mathbb{A}$ is uniformly bounded on any bounded subset $\Omega_{0}$ of $E$. Now, for $u \in \Omega_{0}$ and $t_{1}, t_{2} \in \mathrm{I}$ such that $t_{1}<t_{2}$, consider

$$
\begin{aligned}
& \left|\mathbb{A} u\left(t_{2}\right)-\mathbb{A} u\left(t_{1}\right)\right| \\
& \leq \int_{0}^{1}\left(\sum_{1}^{m}\left|K_{\beta_{i}}\left(s, t_{2}\right)-K_{\beta_{i}}\left(s, t_{1}\right)\right|\left|h_{i}\left(s, u, \mathcal{D}^{\omega-1} u(s)\right)\right|\right. \\
& \left.\quad+\left|K_{0}\left(s, t_{2}\right)-K_{0}\left(s, t_{1}\right)\right|\left|\Psi\left(s, u, \mathcal{D}^{\omega-1} u(s)\right)\right|\right) d s, \\
& \left|\mathcal{D}^{\omega-1} \mathbb{A} u\left(t_{2}\right)-\mathcal{D}^{\omega-1} \mathbb{A} u\left(t_{1}\right)\right| \\
& \leq \int_{0}^{1}\left(\sum_{1}^{m}\left|G_{\beta_{i}}\left(s, t_{2}\right)-G_{\beta_{i}}\left(s, t_{1}\right)\right|\left|h_{i}\left(s, u, \mathcal{D}^{\omega-1} u(s)\right)\right|\right. \\
& \left.\quad+\left|G_{0}\left(s, t_{2}\right)-G_{0}\left(s, t_{1}\right)\right|\left|\Psi\left(s, u, \mathcal{D}^{\omega-1} u(s)\right)\right|\right) d s .
\end{aligned}
$$

But

$$
\begin{aligned}
& \left|K_{\beta_{i}}\left(s, t_{2}\right)-K_{\beta_{i}}\left(s, t_{1}\right)\right| \\
& =\frac{1}{\Gamma\left(\omega+\beta_{i}\right)} \begin{cases}(1-s)^{\omega+\beta_{i}-1}\left(t_{2}-t_{1}\right) ; & t \leq s, \\
(1-s)^{\omega+\beta_{i}-1}\left(t_{2}-t_{1}\right)+\left(t_{2}-s\right)^{\omega-1+\beta_{i}}-\left(t_{1}-s\right)^{\omega-1+\beta_{i}} ; & s \leq t,\end{cases} \\
& \left|K_{0}\left(s, t_{2}\right)-K_{0}\left(s, t_{1}\right)\right|=\frac{1}{\Gamma(\omega)} \begin{cases}(1-s)^{\omega-1}\left(t_{2}-t_{1}\right) ; & t \leq s, \\
(1-s)^{\omega-1}\left(t_{2}-t_{1}\right)+\left(t_{2}-s\right)^{\omega-1}-\left(t_{1}-s\right)^{\omega-1} ; & s \leq t,\end{cases} \\
& \left|G_{\beta_{i}}\left(s, t_{2}\right)-G_{\beta_{i}}\left(s, t_{1}\right)\right|
\end{aligned}
$$




$$
\begin{gathered}
=\frac{1}{\Gamma \omega \Gamma\left(\omega+\beta_{i}\right)} \begin{cases}(1-s)^{\omega+\beta_{i}-1}\left(t_{2}^{2-\omega}-t_{1}^{2-\omega}\right) ; & t \leq s, \\
(1-s)^{\omega+\beta_{i}-1}\left(t_{2}^{2-\omega}-t_{1}^{2-\omega}\right)+\left(t_{2}-s\right)^{\beta_{i}}-\left(t_{1}-s\right)^{\beta_{i} ;} & s \leq t,\end{cases} \\
\left|G_{0}\left(s, t_{2}\right)-G_{0}\left(s, t_{1}\right)\right|=\frac{1}{\Gamma(\omega) \Gamma(3-\omega)} \begin{cases}(1-s)^{\omega-1}\left(t_{2}^{2-\omega}-t_{1}^{2-\omega}\right) ; & t \leq s, \\
(1-s)^{\omega-1}\left(t_{2}^{2-\omega}-t_{1}^{2-\omega}\right) ; & s \leq t .\end{cases}
\end{gathered}
$$

Hence, using the relation $\int_{0}^{1} a(t, s) d s=\int_{0}^{t} a(t, s) d s+\int_{t}^{1} a(t, s) d s$ and the notation $\Delta F(s, t)=$ $F\left(s, t_{2}\right)-F\left(s, t_{2}\right)$ for the difference, we obtain

$$
\begin{aligned}
& \int_{0}^{1}\left|\Delta K_{\beta_{i}}(s, t)\right| d s=\frac{\left.\left[\left(t_{2}-t_{1}\right)+\left(t_{2}^{\omega+\beta_{i}}-t_{1}^{\omega+\beta_{i}}\right)-\left(t_{2}-t\right)^{\omega+\beta_{i}}+\left(t_{1}-t\right)^{\omega+\beta_{i}}\right)\right]}{\Gamma\left(\omega+1+\beta_{i}\right)}, \\
& \left.\int_{0}^{1}\left|\Delta K_{0}(s, t)\right| d s=\frac{1}{\Gamma(\omega+1)}\left[\left(t_{2}-t_{1}\right)+\left(t_{2}^{\omega}-t_{1}^{\omega}\right)-\left(t_{2}-t\right)^{\omega}+\left(t_{1}-t\right)^{\omega}\right)\right] \\
& \int_{0}^{1}\left|\Delta G_{\beta_{i}}(s, t)\right| d s=\frac{\left.\left[\left(t_{2}^{2-\omega}-t_{1}^{2-\omega}\right)+t_{2}^{\beta_{i}+1}-t_{1}^{\beta_{i}+1}-\left(t_{2}-t\right)^{\beta_{i}+1}+\left(t_{1}-t\right)^{\beta_{i}+1}\right)\right]}{\Gamma(3-\omega) \Gamma\left(\omega+1+\beta_{i}\right)} \\
& \int_{0}^{1}\left|\Delta G_{0}(s, t)\right| d s=\frac{1}{\Gamma(3-\omega) \Gamma(\omega+1)}\left[t_{2}^{2-\omega}-t_{1}^{2-\omega}\right] .
\end{aligned}
$$

Using (21), the assumption $\left|h_{i}\left(s, u, \mathcal{D}^{\omega-1} u(s)\right)\right| \leq\left\|\theta_{i}\right\|$ on $\Omega_{0}$, and (26) in (24) and (25), it follows that

$$
\left|\mathbb{A} u\left(t_{2}\right)-\mathbb{A} u\left(t_{1}\right)\right| \rightarrow 0, \quad\left|\mathcal{D}^{\omega-1} \mathbb{A} u\left(t_{2}\right)-\mathcal{D}^{\omega-1} \mathbb{A} u\left(t_{1}\right)\right| \rightarrow 0 \quad \text { as } t_{1} \rightarrow t_{2}
$$

Therefore $\mathbb{A}$ is equicontinuous, and by Arzela-Ascoli theorem $\mathbb{A}$ is compact. By Proposition 2.3, the operator $\mathbb{A}$ is $\varrho^{* *}$-Lipschitz with zero constant.

Theorem 3.1 Under assumptions $\left(H_{1}\right)-\left(H_{3}\right)$, system (13) has at least one solution $u \in E$ provided that $q \leq 1-\delta, E_{3}<1$. Also, the set of solutions of (13) is bounded in $\mathbb{E}$.

Proof By Lemma 3.2, the operator $\mathbb{B}$ is $\varrho^{* *}$ - Lipschitz for $k \in[0,1)$, and by Lemma 3.3, the operator $\mathbb{A}$ is $\varrho^{* *}$ - Lipschitz with zero constant. It follows by Proposition 2.2 that $\mathbb{T}$ is $\varrho^{* *}$ Lipschitz with constant $k \in[0,1)$. Define

$$
\mathbb{G}=\{u \in \mathbb{E}: \exists \hbar \in[0,1] \text { such that } u=\hbar \mathbb{T} u\} .
$$

For $u \in \mathbb{G}$, using the growth conditions (20) and (23), we obtain

$$
\begin{aligned}
\|u\|_{1} & \leq \hbar\left(\|\mathbb{A} u\|_{1}+\|\mathbb{B} u\|_{1}\right) \\
& \leq \hbar\left(d\|u\|_{1}^{q_{1}}+e+E_{0}+E_{1}\|u\|_{1}^{\delta}+E_{2}\|u\|_{1}^{q}+E_{3}\|u\|_{1}^{q+\delta}\right) \\
& =\hbar\left(d\|u\|_{1}^{q_{1}}+E_{1}\|u\|_{1}^{\delta}+E_{2}\|u\|_{1}^{q}+E_{3}\|u\|_{1}^{q+\delta}\right)+\hbar\left(e+E_{0}\right) .
\end{aligned}
$$

Since $q \leq 1-\delta$ and $E_{3}=\frac{v \varrho^{* *}{ }_{0}}{\Gamma(\omega) \Gamma(\vartheta+1)}\left(1+\frac{1}{\Gamma(3-\omega)}\right)\left(1+\frac{1}{(\Gamma(\gamma+1))^{q}}\right)<1$, it follows that the set $\mathbb{G}$ is bounded. Hence, by Theorem (2.4), BVP (1) has at least one solution.

Choose $0<R<1$ and consider a closed bounded and convex subset $\bar{B}=\left\{z \in E:\|z\|_{1} \leq\right.$ $R\} \subseteq \mathbb{E}$. 
Theorem 3.2 Under assumptions $\left(H_{1}\right)-\left(H_{4}\right)$, system (13) has a unique solution in $\bar{B}$ provided that

$$
\begin{aligned}
k & +\sum_{1}^{m} \frac{\lambda^{* *}{ }_{i}\left(1+\frac{1}{\Gamma(3-\omega)}\right)}{\Gamma\left(\omega+\beta_{i}\right)} \\
& +\frac{\varrho^{* *}{ }_{0}\left(1+\frac{1}{\Gamma(3-\omega)}\right)}{\Gamma(\vartheta+1) \Gamma \omega}\left(\rho_{0}+v\left(1+\frac{1}{(\Gamma(\gamma+1))^{q}}\right) R^{q}+\rho_{0}\left(\varrho^{* *}{ }_{0} R^{\delta}+\xi\right)\right)<1 .
\end{aligned}
$$

Proof For $u \in \bar{B}$, using $H_{3}$, we obtain

$$
\begin{aligned}
& \left|f\left(t, u(t), \mathcal{D}^{\omega-1} u(t)\right)\right| \leq \varrho^{* *}{ }_{0} R^{\delta}+\xi \\
& \left|I^{\vartheta} g\left(t, u(t), I^{\gamma} u(t)\right)\right| \leq \frac{\left(\rho_{0}+v\left(1+\frac{1}{(\Gamma(\gamma+1))^{q}}\right) R^{q}\right)}{\Gamma(\vartheta+1)} .
\end{aligned}
$$

For $u_{1}, u_{2} \in \bar{B}$, using $H_{4}$, we obtain

$$
\begin{aligned}
& \left|h_{i}\left(t, u_{1}(t), \mathcal{D}^{\omega-1} u_{1}(t)\right)-h_{i}\left(t, u_{2}(t), \mathcal{D}^{\omega-1} u_{2}(t)\right)\right| \leq \lambda^{* *}{ }_{i}\left\|u_{1}-u_{2}\right\|_{1}, \\
& \left|f\left(t, u_{1}(t), \mathcal{D}^{\omega-1} u_{1}(t)\right)-f\left(t, u_{2}(t), \mathcal{D}^{\omega-1} u_{2}(t)\right)\right| \leq \varrho^{* *}{ }_{0}\left\|u_{1}-u_{2}\right\|_{1}, \\
& \left|I^{\vartheta} g\left(t, u_{1}(t), I^{\gamma} u_{1}(t)\right)-I^{\vartheta} g\left(t, u_{2}(t), I^{\gamma} u_{2}(t)\right)\right| \leq \frac{\rho_{0}\left|u_{1}-u_{2}\right|}{\Gamma(\vartheta+1)} \leq \frac{\rho_{0}\left\|u_{1}-u_{2}\right\|_{1}}{\Gamma(\vartheta+1)} .
\end{aligned}
$$

Further,

$$
\begin{aligned}
& \left|\Psi\left(t, u_{1}, \mathcal{D}^{\omega-1} u_{1}\right)-\Psi\left(t, u_{2}, \mathcal{D}^{\omega-1} u_{2}\right)\right| \\
& \quad \leq\left|I^{\vartheta} g\left(t, u_{1}, I^{\gamma} u_{1}\right)\right|\left|f\left(t, u_{1}, \mathcal{D}^{\omega-1} u_{1}\right)-f\left(t, u_{2}, \mathcal{D}^{\omega-1} u_{2}\right)\right| \\
& \quad+\left|f\left(t, u_{2}, \mathcal{D}^{\omega-1} u_{2}\right)\right|\left|I^{\vartheta} g\left(t, u_{1}, I^{\gamma} u_{1}\right)-I^{\vartheta} g\left(t, u_{2}(t), I^{\gamma} u_{2}(t)\right)\right|,
\end{aligned}
$$

which in view of (29) implies that

$$
\begin{aligned}
& \left|\Psi\left(t, u_{1}, \mathcal{D}^{\omega-1} u_{1}\right)-\Psi\left(t, u_{2}, \mathcal{D}^{\omega-1} u_{2}\right)\right| \\
& \quad \leq\left(\frac{\left(\rho_{0}+v\left(1+\frac{1}{(\Gamma(\gamma+1))^{q}}\right) R^{q}\right) \varrho^{* *}{ }_{0}}{\Gamma(\vartheta+1)}+\frac{\left(\varrho^{* *}{ }_{0} R^{\delta}+\xi\right) \rho_{0}}{\Gamma(\vartheta+1)}\right)\left\|u_{1}-u_{2}\right\|_{1} .
\end{aligned}
$$

Now, using definition (12), we obtain

$$
\begin{aligned}
\left\|\mathbb{A}\left(u_{1}\right)-\mathbb{A}\left(u_{1}\right)\right\|_{1} \\
\leq \\
\quad \int_{0}^{1}\left(\sum_{1}^{m}\left|K_{\beta_{i}}(s, t)+G_{\beta_{i}}(s, t)\right| h_{i}\left(s, u_{1}, \mathcal{D}^{\omega-1} u_{1}\right)-h_{i}\left(s, u_{2}, \mathcal{D}^{\omega-1} u_{2}\right) \mid\right. \\
\quad+\left(\left|K_{0}(s, t)+G_{0}(s, t)\right|\right) \mid \Psi\left(s, u_{1}, \mathcal{D}^{\omega-1} u_{1}-\Psi\left(s, u_{2}, \mathcal{D}^{\omega-1} u_{2} \mid\right)\right) d s,
\end{aligned}
$$


which in view of (30) and (31) implies that

$$
\begin{aligned}
\left\|\mathbb{A}\left(u_{1}\right)-\mathbb{A}\left(u_{2}\right)\right\|_{1} \leq & {\left[\sum_{1}^{m} \frac{\lambda^{* *}{ }_{i}\left(1+\frac{1}{\Gamma(3-\omega)}\right)}{\Gamma\left(\omega+\beta_{i}\right)}+\frac{\left(1+\frac{1}{\Gamma(3-\omega)}\right)}{\Gamma(\omega) \Gamma(\vartheta+1)}\left(\varrho ^ { * * } { } _ { 0 } \left(\rho_{0}\right.\right.\right.} \\
& \left.\left.\left.+v\left(1+\frac{1}{(\Gamma(\gamma+1))^{q}}\right) R^{q}\right)+\rho_{0}\left(\varrho^{* *}{ }_{0} R^{\delta}+\xi\right)\right)\right]\left\|u_{1}-u_{2}\right\|_{1} \\
= & k_{1}\left\|u_{1}-u_{2}\right\|_{1},
\end{aligned}
$$

where

$$
\begin{aligned}
k_{1}= & \sum_{1}^{m} \frac{\lambda^{* *}{ }_{i}\left(1+\frac{1}{\Gamma(3-\omega)}\right)}{\Gamma\left(\omega+\beta_{i}\right)} \\
& +\frac{\left(1+\frac{1}{\Gamma(3-\omega)}\right)}{\Gamma(\omega) \Gamma(\vartheta+1)}\left(\varrho^{* *}{ }_{0}\left(\rho_{0}+v\left(1+\frac{1}{(\Gamma(\gamma+1))^{q}}\right) R^{q}\right)+\rho_{0}\left(\varrho^{* *}{ }_{0}^{\delta}+\xi\right)\right) .
\end{aligned}
$$

Hence, using (17) and (32), it follows that

$$
\begin{aligned}
\left\|\mathbb{T}\left(u_{1}\right)-\mathbb{T}\left(u_{2}\right)\right\|_{1} & \leq\left\|\mathbb{A}\left(u_{1}\right)-\mathbb{A}\left(u_{2}\right)\right\|_{1}+\left\|\mathbb{B}\left(u_{1}\right)-\mathbb{B}\left(u_{2}\right)\right\|_{1} \\
& \leq\left(k+k_{1}\right)\left\|u_{1}-u_{2}\right\|_{1},
\end{aligned}
$$

and uniqueness follows by the Banach contraction principle.

\section{Hyers-Ulam stability}

In this section, we present the Hyers-Ulam stability analysis for the hybrid fractional differential equation (1). For more related problems to the Hyers-Ulam stability, the readers may take help from the references in [15-20] and the literature.

Definition 4.1 The fractional integral system (13) is said to be Hyers-Ulam stable if there exists a constant $\zeta>0$ such that, for given $\varphi>0$ and for each solution $u$ of the inequality

$$
\|u(t)-(\mathbb{A}+\mathbb{B}) u(t)\|_{1}<\varphi,
$$

there exists a solution $\bar{u}(t)$ of the integral system (13)

$$
\bar{u}(t)=(\mathbb{A}+\mathbb{B}) \bar{u}(t)
$$

such that

$$
\|u(t)-\bar{u}(t)\|_{1}<\varphi \zeta
$$

Theorem 4.2 Under assumptions $\left(H_{2}\right)$ and $\left(H_{4}\right)$, the fractional order hybrid differential equation (1) is Hyers-Ulam stable provided $k+k_{1}<1$. 
Proof Let $u \in E$ satisfy inequality (34) and $\bar{u} \in E$ be a solution of BVP (1) satisfying the integral system (13). Then consider

$$
\begin{aligned}
\|u(t)-\bar{u}(t)\|_{1}= & \|u(t)-(\mathbb{A}+\mathbb{B}) \bar{u}(t)\|_{1} \\
\leq & \|u(t)-(\mathbb{A}+\mathbb{B}) u(t)\|_{1} \\
& +\|(\mathbb{A}+\mathbb{B}) u(t)-(\mathbb{A}+\mathbb{B}) \bar{u}(t)\|_{1} \\
< & \varphi+\|(\mathbb{A}+\mathbb{B}) u(t)-(\mathbb{A}+\mathbb{B}) \bar{u}(t)\|_{1} .
\end{aligned}
$$

Now

$$
\|(\mathbb{A}+\mathbb{B}) u(t)-(\mathbb{A}+\mathbb{B}) \bar{u}(t)\|_{1} \leq\|\mathbb{A} u(t)-\mathbb{A} \bar{u}(t)\|_{1}+\|\mathbb{B} u(t)-\mathbb{B} \bar{u}(t)\|_{1},
$$

which in view of $H_{2}$ and $H_{4}$ (that is, (17) and (32)) implies that

$$
\|(\mathbb{A}+\mathbb{B}) u(t)-(\mathbb{A}+\mathbb{B}) \bar{u}(t)\|_{1} \leq k_{1}\|u(t)-\bar{u}(t)\|_{1}+k\|u(t)-\bar{u}(t)\|_{1} .
$$

Hence, from (35), it follows that

$$
\|u(t)-\bar{u}(t)\|_{1}<\varphi+\left(k_{1}+k\right)\|u(t)-\bar{u}(t)\|_{1}
$$

which implies that

$$
\|u(t)-\bar{u}(t)\|_{1}<\varphi \zeta, \quad \text { where } \zeta=\frac{1}{1-\left(k_{1}+k\right)} .
$$

\section{Application}

In this section, we present an example in the application of the results we studied in the previous sections.

Example 1 We consider

$$
\begin{aligned}
& { }^{c} \mathcal{D}^{\vartheta}\left[\frac{{ }^{c} \mathcal{D}^{\omega} u(t)-\sum_{1}^{m} I^{\beta_{i}} h_{i}\left(t, u(t), \mathcal{D}^{\omega-1} u(t)\right)}{f\left(t, u(t), \mathcal{D}^{\omega-1} u(t)\right)}\right]=g\left(t, u(t), I^{\gamma} u(t)\right), \quad t \in \mathrm{I}=[0,1] \\
& { }^{c} \mathcal{D}^{\omega} u(0)=0, \quad u(0)=\psi_{1}\left(u\left(\zeta_{0}\right)\right), \quad u(1)=\psi_{2}\left(u\left(\zeta_{0}\right)\right),
\end{aligned}
$$

where the parameters are such that $0<\vartheta \leq 1,1<\omega \leq 2,0<\zeta_{0}<1$, the functions $f:$ I $\times$ $\mathcal{R}_{e} \times \mathcal{R}_{e} \rightarrow \mathcal{R}_{e}-\{0\}, h_{i}: \mathrm{I} \times \mathcal{R}_{e} \times \mathcal{R}_{e} \rightarrow \mathcal{R}_{e}(i=1,2, \ldots, m)$, and $g: \mathrm{I} \times \mathcal{R}_{e} \times \mathcal{R}_{e} \rightarrow \mathcal{R}_{e}$ such that $\vartheta=0.5, \omega=1.5, \zeta_{0}=0.5, \beta_{i}=0.5$ for $i=1,2, \ldots, m . q=0.2, \delta=0.7$ and $\psi_{1}(u(t))=$ $\psi_{2}(u(t))=\frac{2+\sin (u(t))}{20}, k_{1}=k_{2}=\frac{1}{10}, h_{i}\left(t, u(t), D^{\omega-1} u(t)\right)=\frac{1+u(t)+D^{\omega-1} u(t)}{50}=f i\left(t, u(t), D^{\omega-1} u(t)\right)$, $g\left(t, u(t), I^{\gamma} u(t)\right)=\frac{1+t+u(t) I^{\gamma} u(t)}{20}$. It is easy to see that $\lambda^{* *}{ }_{i}=\frac{1}{50}$ for $i=1,2, \ldots, m, \rho=1+t$, $v=\frac{1}{20}$. And

$$
\begin{aligned}
& K_{\beta_{i}}(s, t)=\frac{-1}{\Gamma\left(\omega+\beta_{i}\right)} \begin{cases}t(1-s)^{\omega-1+\beta_{i} ;} & t \leq s, \\
t(1-s)^{\omega-1+\beta_{i}}-(t-s)^{\omega-1+\beta_{i} ;} & s \leq t,\end{cases} \\
& K_{0}(s, t)=\frac{-1}{\Gamma(\omega)} \begin{cases}t(1-s)^{\omega-1} ; & t \leq s, \\
t(1-s)^{\omega-1}-(t-s)^{\omega-1} ; & s \leq t .\end{cases}
\end{aligned}
$$


It is easy to see that $\left(H_{1}\right)-\left(H_{4}\right)$ are satisfied, also the inequality

$$
\begin{aligned}
k & +\sum_{1}^{m} \frac{\lambda^{* *}{ }_{i}\left(1+\frac{1}{\Gamma(3-\omega)}\right)}{\Gamma\left(\omega+\beta_{i}\right)} \\
& +\frac{\varrho^{* *}{ }_{0}\left(1+\frac{1}{\Gamma(3-\omega)}\right)}{\Gamma(\vartheta+1) \Gamma \omega}\left(\rho_{0}+v\left(1+\frac{1}{(\Gamma(\gamma+1))^{q}}\right) R^{q}+\rho_{0}\left(\varrho^{* *}{ }_{0} R^{\delta}+\xi\right)\right)<1,
\end{aligned}
$$

holds true. Thus, problem (37) has a unique solution. For more applications of the results, we refer the readers to the work in [21-29].

\section{Conclusion}

In this article, we have studied a general class of hybrid fractional differentials for the existence, uniqueness, and Hyers-Ulam stability. We have seen that under certain assumptions of $\left(H_{1}\right)-\left(H_{4}\right)$, the FDEs of the kind (1) have unique solutions and they are Hyers-Ulam stable too, subject to the inequalities given in the statements. At the end, we also presented an example as an application of the work. We suggest the readers for re-consideration of the suggested problem for the $\mathrm{ABC}$-fractional order derivative and others too.

\section{Acknowledgements}

All the authors are thankful to the reviewers and the editor for their comments which have improved the quality of the article.

Funding

There is no source of funding this article.

\section{Availability of data and materials}

Not applicable.

Competing interests

The authors declare that they have no competing interests.

\section{Authors' contributions}

All the authors have equal contributions in this article. All authors read and approved the final manuscript.

\section{Author details}

${ }^{1}$ Department of Mathematics, University of Malakand, Chakadara Dir(L), Khyber Pakhtunkhwa, Pakistan. ${ }^{2}$ Department of Mathematics, Shaheed Benazir Bhutto University, Sheringal Dir Upper, Khyber Pakhtunkhwa, Pakistan. ${ }^{3}$ Department of Mathematics, Cankaya University, Ankara, Turkey. ${ }^{4}$ Department of Medical Research, China Medical University Hospital, China Medical University, Taichung, Taiwan.

\section{Publisher's Note}

Springer Nature remains neutral with regard to jurisdictional claims in published maps and institutional affiliations.

Received: 24 March 2021 Accepted: 31 May 2021 Published online: 11 June 2021

\section{References}

1. Zhao, Y., Sun, S., Han, Z., Li, Q.: Theory of fractional hybrid differential equations. Comput. Math. Appl. 62(3), 1312-1324 (2011)

2. Sun, S., Zhao, Y., Han, Z., Li, Y.: The existence of solutions for boundary value problem of fractional hybrid differential equations. Commun. Nonlinear Sci. Numer. Simul. 17(12), 4961-4967 (2012)

3. Ahmad, B., Ntouyas, S.K.: An existence theorem for fractional hybrid differential inclusions of Hadamard type with Dirichlet boundary conditions. Abstr. Appl. Anal. 2014, Article ID 705809 (2014)

4. Dhage, B.C., Ntouyas, S.K.: Existence results for boundary value problems for fractional hybrid differential inclusions. Topol. Methods Nonlinear Anal. 44(1), 229-238 (2014)

5. Ahmad, B., Ntouyas, S.K., Alsaedi, A.: Existence results for a system of coupled hybrid fractional differential equations. Sci. World J. 2014, Article ID 426438 (2014)

6. Dhage, B.C.: Quadratic perturbations of periodic boundary value problems of second order ordinary differential equations. Differ. Equ. Appl. 2(4), 465-486 (2010) 
7. Dhage, B.C.: Periodic boundary value problems of first order Caratheodory and discontinuous differential equations. Nonlinear Funct. Anal. Appl. 13(2), 323-352 (2008)

8. Dhage, B.: Basic results in the theory of hybrid differential equations with mixed perturbations of second type. Funct. Differ. Equ. 19, 1-20 (2012)

9. Sitho, S., Ntouyas, S.K., Tariboon, J.: Existence results for hybrid fractional integro-differential equations. Bound. Value Probl. 2015, 113 (2015)

10. Hannabou, M., Hilal, K., Kajouni, A.: Existence results of hybrid fractional sequential integro-differential equations. Eng. Math. Lett. 2, 1-19 (2020)

11. Jamil, M., Khan, R.A., Shah, K.: Existence theory to a class of boundary value problems of hybrid fractional sequential integro-differential equations. Bound. Value Probl. 2019, 77 (2019)

12. Kuratowski, K:: Topologie, Warszawa (1952)

13. Deimling, K.: Nonlinear Functional Analysis. Springer, New York (1985)

14. Isaia, F:. On a nonlinear integral equation without compactness. Acta Math. Univ. Comen. 2, 233-240 (2006)

15. Shah, A., Khan, R.A., Khan, A., Khan, H., Gomez-Aguilar, J.F.: Investigation of a system of nonlinear fractional order hybrid differential equations under usual boundary conditions for existence of solution. Math. Methods Appl. Sci. 44(2), 1628-1638 (2021)

16. Khan, A., Abdeljawad, T., Shatanawi, W., Khan, H.: Fixed point theorems for quadruple self-mappings satisfying integral type inequalities. Filomat 34(3), 905-917 (2020)

17. Ahmad, S., Ullah, A., Al-Mdallal, Q.M., Khan, H., Shah, K., Khan, A.: Fractional order mathematical modeling of COVID-19 transmission. Chaos Solitons Fractals 139, 110256 (2020)

18. Shah, K., Khan, Z.A., Ali, A., Amin, R., Khan, H., Khan, A.: Haar wavelet collocation approach for the solution of fractional order COVID-19 model using Caputo derivative. Alex. Eng. J. 59(5), 3221-3231 (2020)

19. Shah, K., Khan, R.A., Khan, A., Khan, H., Gomez-Aguilar, J.F.: Investigation of a system of nonlinear fractional order hybrid differential equations under usual boundary conditions for the existence of solution. Math. Methods Appl. Sci. 44(2), 1628-1638 (2020). https://doi.org/10.1002/mma.6865

20. Khan, H., Khan, Z.A., Tajadodi, H., Khan, A.: Existence and data-dependence theorems for fractional impulsive integro-differential system. Adv. Differ. Equ. 458, 1-11 (2020)

21. Baleanu, D., Etemad, S., Rezapour, S.: A hybrid Caputo fractional modeling for thermostat with hybrid boundary value conditions. Bound. Value Probl. 2020, 1 (2020)

22. Baleanu, D., Etemad, S., Rezapour, S.: On a fractional hybrid integro-differential equation with mixed hybrid integral boundary value conditions by using three operators. Alex. Eng. J. 59(5), 3019-3027 (2020)

23. Rezapour, S., Imran, A., Hussain, A., Martinez, F., Etemad, S., Kaabar, M.K.: Condensing functions and approximate endpoint criterion for the existence analysis of quantum integro-difference FBVPs. Symmetry 13(3), 469 (2021)

24. Rezapour, S., Chikh, S.B., Amara, A., Ntouyas, S.K., Tariboon, J., Etemad, S.: Existence results for Caputo-Hadamard nonlocal fractional multi-order boundary value problems. Mathematics 9(7), 719 (2021)

25. Baleanu, D., Jajarmi, A., Mohammadi, H., Rezapour, S.: A new study on the mathematical modelling of human liver with Caputo-Fabrizio fractional derivative. Chaos Solitons Fractals 134, 109705 (2020)

26. Tuan, N.H., Mohammadi, H., Rezapour, S.: A mathematical model for COVID-19 transmission by using the Caputo fractional derivative. Chaos Solitons Fractals 140, 110107 (2020)

27. Mohammadi, H., Kumar, S., Rezapour, S., Etemad, S.: A theoretical study of the Caputo-Fabrizio fractional modeling for hearing loss due to Mumps virus with optimal control. Chaos Solitons Fractals 144, 110668 (2021)

28. Rezapour, S., Ntouyas, S.K., labal, M.Q., Hussain, A., Etemad, S., Tariboon, J.: An analytical survey on the solutions of the generalized double-order-integrodifferential equation. J. Funct. Spaces 2021, Article ID 6667757 (2021)

29. Baleanu, D., Etemad, S., Rezapour, S.: A hybrid Caputo fractional modeling for thermostat with hybrid boundary value conditions. Bound. Value Probl. 2020, 64 (2020)

\section{Submit your manuscript to a SpringerOpen ${ }^{\circ}$ journal and benefit from:}

- Convenient online submission

- Rigorous peer review

- Open access: articles freely available online

- High visibility within the field

- Retaining the copyright to your article

Submit your next manuscript at $\gg$ springeropen.com 